\title{
The emerging role of bexarotene in the treatment of Alzheimer's disease: current evidence
}

This article was published in the following Dove Press journal:

Neuropsychiatric Disease and Treatment

5 February 2015

Number of times this article has been viewed

\author{
Babak Tousi \\ Cleveland Clinic Lou Ruvo Center \\ for Brian Health, Neurological \\ Institute, Cleveland, OH, USA
}

\begin{abstract}
In 2012, a novel approach to the treatment of Alzheimer's disease was introduced, heralding a wave of excitement in the field of dementia. Bexarotene, a retinoid X receptor agonist, was shown to reverse neurodegeneration, improve cognition, and decrease levels of amyloid- $\beta$ in transgenic mice expressing familial Alzheimer disease mutations. Since then, there has been widespread discussion about bexarotene, as well as a number of follow-up studies. Bexarotene is a unique compound, as it is approved by the US Food and Drug Administration for other purposes and there are reasonable data to justify its mechanism of action in dementia. This review discusses these studies and the emerging role of bexarotene in the clinical field of Alzheimer's dementia.
\end{abstract}

Keywords: repurposing, ApoE-targeted mice, amyloid, therapy, dementia

\section{Introduction}

Our knowledge about the pathogenesis of Alzheimer's dementia (AD) has expanded dramatically over the last decade. Unfortunately, these findings have not been successfully translated into new therapies for AD. These attempts by the pharmaceutical industry to find new treatments have not been successful during the last decade, with an overall failure rate of $99.6 \%{ }^{1}$ and only one newly approved therapy since 2004 in the United States.

The pharmaceutical industry has scaled down the search for new Alzheimer's treatments after the failure of several high-profile expensive trials. This, however, might have initiated a trend of investigating drugs that have already been approved by the US Food and Drug Administration for other indications, but that may interact with $\mathrm{AD}$ pathophysiology through mechanisms unrelated to their original therapeutic indication. Because these medications have already gone through Phase I and Phase II trials, their chances of passing through additional trials, if necessary, are very good. That by itself facilitates progression through the clinical trial pipeline, ${ }^{1}$ which can save years of work and millions of dollars.

A landmark paper by Landreth and Cramer et al published in Science on bexarotene, a compound currently approved to treat cutaneous T-cell lymphomas, created potential new hope for the treatment of $\mathrm{AD}{ }^{2}{ }^{2}$ This review describes and discusses the literature addressing the effects of bexarotene in an Alzheimer's model of mice and the potential clinical implications.

\section{Background}

Disease-modifying therapies can be classified mainly as amyloid-related, tau-related, and neuroprotective. The therapies that target amyloid processing in Alzheimer's

$\mathrm{Tel}+\mathrm{I} 2165212228$

Email babak.tousi@ccf.org 
disease rely on different mechanisms of action: inhibition of beta secretase or gamma secretase, enhancement of alpha secretase or amyloid- $\beta(A \beta)$ metabolism, and even amyloid removal by monoclonal antibodies or plasma exchange. ${ }^{3,4}$

Increased production and/or decreased clearance of $\mathrm{A} \beta$ from the brain can contribute to Alzheimer's disease. Therapeutic strategies to promote $A \beta$ clearance, such as the use of apolipoprotein E (APO E), hold promise for the treatment of $\mathrm{AD}$.

\section{APO E and Alzheimer's disease}

APO $\mathrm{E}$ is a lipid transport protein that promotes $\mathrm{A} \beta$ degradation and protects against atherosclerosis. APO E exists in three isoforms: E2, E3, and E4. Most humans are homozygous for APO E3. APO E4 is considered a risk factor for late-onset $\mathrm{AD}$, and $\mathrm{APO} \mathrm{E} 2$ is considered protective. ${ }^{5}$

Expression of APOE and the lipid transporter genes $A B C A 1$ and $A B C G 1$ facilitate the formation of APO E-associated lipoprotein particles. ${ }^{6}$ This is regulated by ligation of either member of the following pair of nuclear receptors: retinoid $\mathrm{X}$ receptor (RXR) and peroxisome proliferator-activated receptors ${ }^{7}$ and RXR and liver X receptor. ${ }^{8}$

Activation of either pair induces the expression of APO E, which promotes A $\beta$ degradation. Agonists of these receptors also promote phagocytosis in microglia and macrophages. ${ }^{9,10}$

\section{Bexarotene in animal models of $A D$}

Landreth and Cramer et al reported that a drug that activates RXRs, such as bexarotene, has the potential to increase APO $\mathrm{E}$ and $\mathrm{ABCA} 1$ production. In their study, bexarotene rapidly cleared soluble $A \beta$ from the brain, reduced neuritic plaque burden, reversed behavioral deficits, and improved cognition in mice genetically engineered to express a mutant form of the $A P P$ gene. ${ }^{2}$

Their impressive results captured worldwide attention and led to additional research. Four studies in particular ${ }^{1-14}$ attempted to replicate and validate Landreth and Cramer's observations that bexarotene stimulates the clearance of soluble $A \beta$ peptides and reverses behavioral deficits in mouse models of Alzheimer's disease. Science published these four follow-up studies as commentary to the original study in a single issue.

Fitz et al found that oral administration of bexarotene reversed cognition in two different tasks to the level of nontransgenic controls. ${ }^{11}$ They also confirmed that soluble $A \beta$ is present in the interstitial fluid. Veeraraghavalu et al also found that oral bexarotene given over the course of 7 days reduced $\mathrm{A} \beta 40$ levels. ${ }^{14}$ Both the Fitz et al and Veeraraghavalu et al studies failed to replicate the effect of amyloid deposition in the brain. ${ }^{11,14}$

Tesseur et al found improvement in social recognition and another memory task, but no change in soluble $A \beta 40$ levels or amyloid deposition after 19 days of treatment. ${ }^{13}$ Price et al were unable to show any reduction in soluble $A \beta 40$ or amyloid deposition. However, the last two studies reported significant upregulation of ABCA1 levels. ${ }^{12}$

In responding to these four follow-up studies, Landreth et $\mathrm{l}^{15}$ emphasized that the additional research validated their main conclusion: bexarotene stimulates clearance of soluble A $\beta$. Only two of the studies, however, replicated most of the original findings. Landreth also emphasized that bexarotene was less efficient in older mice or in more aggressive amyloidogenic models of mice, which might have explained why these follow-up studies failed to find any reductions in amyloid plaques. They concluded, "the behavioral improvements were poorly correlated with the microglial-mediated removal of insoluble, deposited forms of $A \beta .{ }^{, 15}$ In addition, they pointed out that the Price et al, ${ }^{12}$ Tesseur et $\mathrm{al}^{13}$ and Veeraraghavalu et $\mathrm{al}^{14}$ studies used unconventional formulations of bexarotene, which alter its pharmacokinetics and bioavailability, a critical difference from the original 2012 study. ${ }^{15}$

Another study, published in an open-access journal, assessed the effects of bexarotene in mutant APP/PS1 mice, which were acutely dosed with the compound. Although ABCA1 was upregulated in the treated mice, there was no attenuation of $A \beta$ plaques or cognitive deficits. ${ }^{16}$

In the latest in vivo study, Boehm-Cagan and Michaelson investigated the effects of bexarotene on messenger RNA and protein levels of APO E, ABCA1, and ABCG1 in young mice and assessed the extent to which this reversed the APO E4-driven pathological phenotype. ${ }^{17}$ This investigation showed that bexarotene increased messenger RNA and protein levels of ABCA1 and ABCG1 in hippocampal neurons. The compound also reversed APO E4-induced cognitive and neuronal impairments. The authors suggested that "RXR activation and increased levels of ABCA1 and ABCG1 could be useful in the treatment of human apoE4 carriers." ${ }^{17}$

In a more recent study, Tai et al ${ }^{18}$ studied the role of the RXR agonist (bexarotene) in EFAD transgenic mice (transgenic mice expressing $5 \times$ familial AD mutations and human APO E3 or APO E4), which have the highest levels of soluble $A \beta$ in E4FAD hippocampus. In E4FAD hippocampus, short-term (6 months) bexarotene treatment increased ABCA1, APO E4 lipoprotein-association/lipidation, and APO E4/A $\beta$ complex 
and decreased soluble $A \beta$ levels. The authors conclude that RXR agonists address the loss of function associated with APO E4 and exacerbated by $A \beta$ pathology.

The above studies may support one of the possible mechanisms of bexarotene's action via amyloid cascade, but there may be other potential mechanisms as well. In vitro studies suggest that APO E4 may affect tau hyperphosphorylation via direct interaction with either tau or other pathways. ${ }^{19,20}$

Further research is required to determine which one of these possible mechanisms underlies the effects of APO E4 on tau hyperphosphorylation in targeted replacement (TR) mice, and its reversal by bexarotene treatment.

\section{Bexarotene in current clinical practice}

Bexarotene is a retinoid approved by the US Food and Drug Administration in 1999 for use as a treatment for cutaneous T-cell lymphoma (CTCL). ${ }^{21}$ Bexarotene is convenient because the capsules can be taken orally once daily with meals. When used to treat cutaneous T-cell lymphoma, the drug can cause adverse effects including hypertriglyceridemia, hypercholesterolemia, and hypothyroidism; these require monitoring but can be managed with concomitant medication. ${ }^{22-24}$

Hypothyroidism caused by bexarotene use was associated with marked reductions in serum concentrations of thyroidstimulating hormone and thyroxine. Bexarotene may suppress thyroid-stimulating hormone production and increase thyroid hormone metabolic clearance. ${ }^{23}$ Treatment of patients with bexarotene-induced hypothyroidism commonly requires high doses of thyroid hormone for replacement therapy, often at twice the typical dose used to treat more common hypothyroidism. ${ }^{22}$

Hypertriglyceridemia developed in $79 \%$ of patients who took bexarotene in a study of early-stage cutaneous T-cell lymphoma, whereas hypercholesterolemia occurred in $48 \%$ of patients, and hypothyroidism in $40 \%{ }^{24}$

Associated hypertriglyceridemia secondary to bexarotene treatment can be managed with medication such as Fenofibrate. There are case series showing that concomitant use of omega-3 fatty acids may adequately manage hypertriglyceridemia in these patients. ${ }^{25}$

\section{Bexarotene in human clinical trials}

Bexarotene is a good candidate for further drug development in AD treatment, given its high blood-brain barrier permeability and current safety profile. However, the pharmaceutical industry might be less enthusiastic in developing a drug that will soon be available generically. Eisai Pharmaceutical has manufactured bexarotene exclusively, and its patent on
Targretin is estimated to expire in $2016 .{ }^{26}$ A generic version may be released by Mylan Inc., in 2015. ${ }^{27}$

Bexarotene has been used off-label in dementia patients since the initial 2012 report, but these cases have not been consistently published. In a single case report of bexarotene use in an APO E4-positive patient with AD, the drug was tolerated relatively well (the most common adverse effect was hypercholesterolemia, which responded to statin therapy), but no cognitive benefit was noted after 3 months of $150 \mathrm{mg}$ daily treatment. ${ }^{28}$

There are currently two clinical studies on human subjects in different phases. The first one, Bexarotene Anti-Amyloid Therapy for Alzheimer's Disease (BEAT-AD), which is being conducted by Dr. Cummings at the Cleveland Clinic, has completed recruiting, and the results are expected to be released this year (Jeffrey Cummings, Cleveland Clinic, 2014). The purpose of this study is to determine the safety and effect of $300 \mathrm{mg}$ bexarotene administered daily for 1 month compared with placebo. The primary study endpoint is the change from baseline to week 4 in amyloid burden, as measured by standard uptake units regional on amyloid brain imaging obtained through $18 \mathrm{~F}-\mathrm{AV}-45$ positron emission tomography. Secondary outcome measures include Alzheimer's Disease Assessment Scale-Cognition change from baseline to week 4 and to week 8, biomarker changes, magnetic resonance imaging changes, Clinical Dementia Rating-Sum of Boxes, Neuropsychiatric Inventory, and Alzheimer's Disease Cooperative Study-Activities of Daily Living Inventory. ${ }^{29}$

Landreth and his laboratory have also developed a trial of bexarotene in healthy people to establish how the medication works in normal subjects. Landreth and Cramer have formed a spinoff company, ReXceptor, to perform the testing. Their primary objective is to determine whether bexarotene enhances the clearance of newly generated $A \beta$. The researchers will also assess whether the drug elevates levels of APO E and APO E-based high density lipoproteins (HDLs) in cerebrospinal fluid (personal communication). ${ }^{30}$

ReXceptor is working in collaboration with $\mathrm{C} 2 \mathrm{~N}$ diagnostics (St Louis, Missouri), which produces a proprietary stable isotope labeling platform, to measure the metabolism of both brain-derived A $\beta$ and APO E (personal communication).

\section{Discussion}

Since the publication of the four follow-up studies on bexarotene, there has been much controversy surrounding the drug's effects on AD. On one hand, the follow-up studies failed to replicate the plaque-clearing finding, which raises 
the concern that it may be too soon to test the drug in patients with Alzheimer's disease. On the other hand, the most important result of the 2012 study, cognitive improvement, has been independently validated. That triggers a new thought process, in that it may be irrelevant if the drug actually clears the plaques and that perhaps our focus needs to shift toward the effects of soluble A $\beta$. Landreth also emphasized that different formulations of bexarotene were used in the validating studies, which may explain the discrepancies between the initial and follow-up study results. ${ }^{15}$

A number of questions remain, however, and these must be addressed in future research. First, how does bexarotene improve cognition? It may do so through an additional mechanism not fully known yet. A French study showed that cholesterol and bexarotene compete for the same binding site in the C-terminal region of Alzheimer's A $\beta 1-42$. Bexarotene competed with cholesterol for binding to $A \beta$ and prevented neurotoxic oligomeric channel formation. ${ }^{31}$ In a recent study, bexarotene was shown to counteract both $\mathrm{A} \beta$-induced and $\mathrm{A} \beta$-independent increases in cortical network hyperexcitability. ${ }^{32}$

Next, how much reduction in $A \beta$ synthesis needs to occur to produce a cognitive benefit?

Third, what is the peripheral effect of bexarotene? We know it noticeably changes the metabolism of lipids in the peripheral blood. Cumming's study at the Cleveland Clinic may shed further light on the effect of bexarotene in Alzheimer's patients. The study has enrolled 20 subjects (10 patients in each group), but whether the study is sufficiently powered to detect efficacy remains to be seen, especially if changes are not as dramatic as those reported in the Landreth mouse model.

Fourth, what is the appropriate dosage for use in a clinical trial of AD? When used to treat cutaneous lymphoma, bexarotene is calculated per milligram per square meter. The dose, however, may be different for the treatment of $\mathrm{AD}$, as the medication must pass through the blood-brain barrier. In addition, it is not clear at what stage of the disease the drug should be given to maximize the effect. If given too late in the disease process, it may not produce the same effects.

What is bexarotene's risk-benefit ratio when used as an Alzheimer's drug? The relatively common adverse effects observed with bexarotene use in cancer patients, such as hyperlipidemia, might add an unjustifiable toxicity burden to a frail patient. Adding other medications (ie, statins) to manage these symptoms might add unexpected pharmacodynamic interactions that obscure any functional effect on cognition. Adverse effects, however, may be overcome by designing an improved analog.
Will the results of the studies of bexarotene treatment using mouse models translate to humans? It is important to emphasize that most published studies have tested bexarotene in transgenic mice expressing mouse APO E, ${ }^{2,12-14}$ which is structurally and functionally distinct from any of the human isoforms. Also, these mouse models failed to develop tauopathy, which is a hallmark finding of the disease in humans.

Will increasing APO E4 produce positive or negative effects? Because bexarotene was shown to increase levels of mouse APO E and ABCA1/ABCG1-induced APO E lipoprotein association/lipidation, it is expected that its effects will depend on the APO E genotype. Accordingly, in the case of APO E3 or mouse APO E, the effects could be beneficial either because of increased levels of APO E and/or because of increased lipidation. However, in the case of APO E4, the effects of increasing APO E4 could be either "good" or "bad," depending on whether APO E4 functions via a toxic gain of function or loss of function.

Finally, we know that bexarotene can address a loss of function associated with APO E4, but is this pathway relevant for APO E3 carriers? Also, is bexarotene an APO E4-specific AD therapy?

Much research remains to be done, but even if bexarotene is found to be ineffective as a treatment for Alzheimer's disease in humans, it has opened the door to finding a new treatment approach to the pathology of this disease and, in fact, may one day represent a new class of $\mathrm{AD}$ therapies.

\section{Acknowledgment}

The author thanks Amy Slugg Moore, BA, for her critical reading of the manuscript and editorial support.

\section{Disclosure}

The author reports no conflicts of interest in this work.

\section{References}

1. Cummings JL, Morstorf T, Zhong K. Alzheimer's disease drugdevelopment pipeline: few candidates, frequent failures. Alzheimers Res Ther. 2014;6(4):37.

2. Cramer PE, Cirrito JR, Wesson DW, et al. ApoE-directed therapeutics rapidly clear $\beta$-amyloid and reverse deficits in $\mathrm{AD}$ mouse models. Science. 2012;335(6075):1503-1506.

3. Counts SE, Lahiri DK. Overview of immunotherapy in Alzheimer's disease (AD) and mechanisms of IVIG neuroprotection in preclinical models of AD. Curr Alzheimer Res. 2014;11(7):623-625.

4. Boada M, Ortiz P, Anaya F, Hernández I. Amyloid-targeted therapeutics in Alzheimer's disease: use of human albumin in plasma exchange as a novel approach for Abeta mobilization. Drug News Perspect. 2009; 22(6):325-339.

5. Raber J, Huang Y, Ashford JW. ApoE genotype accounts for the vast majority of AD risk and AD pathology. Neurobiol Aging. 2004;25(5): $641-650$. 
6. Kim WS, Weickert CS, Garner B. Role of ATP-binding cassette transporters in brain lipid transport and neurological disease. J Neurochem. 2008;104(5):1145-1166.

7. Chawla A, Boisvert WA, Lee $\mathrm{CH}$, et al. A PPAR gamma-LXR-ABCA1 pathway in macrophages is involved in cholesterol efflux and atherogenesis. Mol Cell. 2001;7(1):161-171.

8. Liang Y, Lin S, Beyer TP, et al. A liver X receptor and retinoid X receptor heterodimer mediates apolipoprotein E expression, secretion and cholesterol homeostasis in astrocytes. J Neurochem. 2004;88(3):623-634.

9. Laffitte BA, Repa JJ, Joseph SB, et al. LXRs control lipid-inducible expression of the apolipoprotein E gene in macrophages and adipocytes. Proc Natl Acad Sci U S A. 2001;98(2):507-512.

10. Mandrekar-Colucci S, Landreth GE. Nuclear receptors as therapeutic targets for Alzheimer's disease. Expert Opin Ther Targets. 2011; 15(9):1085-1097.

11. Fitz NF, Cronican AA, Lefterov I, Koldamova R. Comment on “ApoEdirected therapeutics rapidly clear $\beta$-amyloid and reverse deficits in $\mathrm{AD}$ mouse models". Science. 2013;340(6135):924-c.

12. Price AR, Xu G, Siemienski ZB, et al. Comment on "ApoE-directed therapeutics rapidly clear $\beta$-amyloid and reverse deficits in AD mouse models". Science. 2013;340(6135):924-d.

13. Tesseur I, Lo AC, Roberfroid A, et al. Comment on "ApoE-directed therapeutics rapidly clear $\beta$-amyloid and reverse deficits in AD mouse models". Science. 2013;340(6135):924-e.

14. Veeraraghavalu K, Zhang C, Miller S, et al. Comment on "ApoEdirected therapeutics rapidly clear $\beta$-amyloid and reverse deficits in AD mouse models". Science. 2013;340(6135):924-f.

15. Landreth GE, Cramer PE, Lakner MM, et al. Response to comments on "ApoE-directed therapeutics rapidly clear $\beta$-amyloid and reverse deficits in AD mouse models". Science. 2013;340(6135):924-g.

16. LaClair KD, Manaye KF, Lee DL, et al. Treatment with bexarotene, a compound that increases apolipoprotein-E, provides no cognitive benefit in mutant APP/PS1 mice. Mol Neurodegener. 2013; $8(1): 18$.

17. Boehm-Cagan A, Michaelson DM. Reversal of apoE4-driven brain pathology and behavioral deficits by bexarotene. J Neurosci. 2014; 34(21):7293-7301.

18. Tai LM, Koster KP, Luo J, et al. Amyloid- $\beta$ Pathology and APOE Genotype Modulate Retinoid X Receptor Agonist Activity in Vivo. J Biol Chem. 2014;289(44):30538-30555.

19. Strittmatter WJ, Saunders AM, Goedert M, et al. Isoform-specific interactions of apolipoprotein E with microtubule-associated protein tau: implications for Alzheimer disease. Proc Natl Acad Sci U S A. 1994; 91(23):11183-11186.
20. Harris FM, Brecht WJ, Xu Q, Mahley RW, Huang Y. Increased tau phosphorylation in apolipoprotein E4 transgenic mice is associated with activation of extracellular signal-regulated kinase: modulation by zinc. J Biol Chem. 2004;279(43):44795-44801.

21. Gniadecki R, Assaf C, Bagot M, et al. The optimal use of bexarotene in cutaneous T-cell lymphoma. Br J Dermatol. 2007;157(3):433-440.

22. Sherman SI. Etiology, diagnosis, and treatment recommendations for central hypothyroidism associated with bexarotene therapy for cutaneous T-cell lymphoma. Clin Lymphoma. 2003;3(4):249-252.

23. Smit JW, Stokkel MP, Pereira AM, Romijn JA, Visser TJ. Bexaroteneinduced hypothyroidism: bexarotene stimulates the peripheral metabolism of thyroid hormones. J Clin Endocrinol Metab. 2007;92(7): 2496-2499.

24. Duvic M, Martin AG, Kim Y, et al; Worldwide Bexarotene Study Group. Phase 2 and 3 clinical trial of oral bexarotene (Targretin capsules) for the treatment of refractory or persistent early-stage cutaneous T-cell lymphoma. Arch Dermatol. 2001;137(5):581-593.

25. Musolino A, Panebianco M, Zendri E, Santini M, Di Nuzzo S, Ardizzoni A. Hypertriglyceridaemia with bexarotene in cutaneous T-cell lymphoma: the role of omega-3 fatty acids. Br J Haematol. 2009;145(1): 84-86.

26. DrugBank. Bexarotene. Available from: http://www.drugbank.ca/drugs/ DB00307. Accessed September 14, 2014.

27. Mylan. Mylan Announces Settlement Agreement for First-to-File Generic Version of TARGRETIN ${ }^{\circledR}$. Canonsburg, PA: Mylan Inc; 2013. Available from: http://investor.mylan.com/releasedetail. cfm?releaseid=814431. Accessed September 14, 2014.

28. Tousi B. Bexarotene for the treatment of cognitive decline in an individual with Alzheimer's dementia. Alzheimer's Dementia. 2013; 9(4):P301.

29. Cleveland Clinic. Bexarotene Amyloid Treatment for Alzheimer's Disease (BEAT-AD). Available from: http://clinicaltrial.gov/ct2/ show/NCT01782742. NLM identifier: NCT01782742. Accessed September 14, 2014.

30. ReXceptor, Inc. A Study to Evaluate the Effect of Bexarotene on Beta-Amyloid and Apolipoprotein E Metabolism in Healthy Subjects. Available from: http:/clinicaltrial.gov/ct2/show/NCT02061878. NLM identifier: NCT02061878. Accessed September 14, 2014.

31. Fantini J, Di Scala C, Yahi N, et al. Bexarotene blocks calciumpermeable ion channels formed by neurotoxic Alzheimer's $\beta$-amyloid peptides. ACS Chem Neurosci. 2014;5(3):216-224.

32. Bomben V, Holth J, Reed J, Cramer P, Landreth G, Noebels J. Bexarotene reduces network excitability in models of Alzheimer's disease and epilepsy. Neurobiol Aging. 2014;35(9):2091-2095.
Neuropsychiatric Disease and Treatment

\section{Publish your work in this journal}

Neuropsychiatric Disease and Treatment is an international, peerreviewed journal of clinical therapeutics and pharmacology focusing on concise rapid reporting of clinical or pre-clinical studies on a range of neuropsychiatric and neurological disorders. This journa is indexed on PubMed Central, the 'PsycINFO' database and CAS,

\section{Dovepress}

and is the official journal of The International Neuropsychiatric Association (INA). The manuscript management system is completely online and includes a very quick and fair peer-review system, which is all easy to use. Visit http://www.dovepress.com/testimonials.php to read real quotes from published authors. 